\title{
Mejoras en la construcción de camisas de cilindro de motores de combustión interna ciclo diese $I^{(\bullet)}$
}

\author{
F. Martínez-Pérez* y A. Barroso-Moreno*
}

\begin{abstract}
Resumen
El análisis del sistema tribológico pistón-segmentos-cilindro durante el funcionamiento de un motor de combustión, permitió valorar las características, mediante el empleo de métodos de ingeniería usados en el mundo, para calcular las tasas de desgaste y otros parámetros resultantes en cada uno de los componentes del sistema antes mencionado. Se definió la camisa de cilindro, como elemento fundamental de los usados en el sistema tribológico y se analizaron las características metalográficas predominantes en su construcción actual de aleaciones de hierro fundido gris con matriz perlítica, lo que permitió proponer una nueva aleación con matriz bainítica que brindara una mayor resistencia al desgaste. Para demostrar el rendimiento de este nuevo producto propuesto se llevó a cabo una técnica experimental basada en el ensayo dinamométrico de un motor de combustión interna ciclo diesel Scania de $150 \mathrm{~kW}$, sometido a plena carga durante $500 \mathrm{~h}$ con $30 \%$ de crecimiento de potencia. Posterior a la prueba, las camisas de cilindro fueron dimensionadas simultáneamente con los segmentos y pistones y como resultado se observó que el material propuesto con una aleación de matriz bainítica tuvo una mejor respuesta al desgaste en comparación con el material de matriz perlítica, inclusive con una mejor estabilidad dimensional. Los segmentos de pistones tuvieron un desgaste similar en ambos materiales. Las conclusiones del trabajo muestran los detalles de los resultados alcanzados durante la investigación.
\end{abstract}

Palabras clave Tribología; Materiales; Desgaste.

\section{Improvement in the production of cylinder shirt of inner diesel combustion engines}

\begin{abstract}
This study deals with the different types of wear as well as other parameters present in the tribological system pistonsegment-cylinder in a combustion engine. By means of engineering methods were defined the wear rates in the three components of the system. The biggest wear in the analysis resulted in the cylinder shirt. Specialized methods applied were used to analyze the prevailing metallographic characteristics in its original construction, obtaining a gray melted iron with perlitic matrix. A new material with bainitic matrix has been proposed for increasing wear resistance. To demonstrate the efficiency of this new product, the experimental techniques carried out, were based on a dynamometric testing in a internal combustion engine diesel cycle Scania of $150 \mathrm{~kW}$. It was exposed to a full charge during $500 \mathrm{~h}$ with $30 \%$ of potency rising. Compared with the perlitic one, it has been proved that the bainitic matrix allows a better result. Besides, a superior dimensional stability was obtained. The piston segments had a similar wear rate in both materials in reference to the original tribological pair of the project.
\end{abstract}

Keywords

Tribology; Materials; Wear.

\section{INTRODUCCIÓN}

Los motores de combustión interna son sistemas cuyas características de funcionamiento causan desgaste, principalmente cuando se piensa en los componentes de la cámara de combustión, como los segmentos o aros, pistones y cilindros o camisas de cilindros. La figura 1 muestra el diseño esquemático de un motor ciclo diesel.
El análisis de las fallas en materiales que pueden causar excesivo desgaste en camisas de cilindros, es más que nunca, de extrema importancia para la industria automovilística e industrial.

De esta forma, las camisas de cilindros de motores de combustión interna, ciclo diesel, demandan un elevado cuidado en el proyecto y en los procesos de fundición, maquinado y otros procesos de elaboración.

(•) Trabajo recibido el día 8 de mayo de 2012 y aceptado en su forma final el día 21 de noviembre de 2012.

* Centro de Estudios de Ingeniería de Mantenimiento, Facultad de Ing. Mecánica, CUJAE. E-mail: fmartinez@ceim.cujae.edu.cu

** Universidad de Pinar del Río, Cuba.E.mail: abarroso@upr.edu.cu 


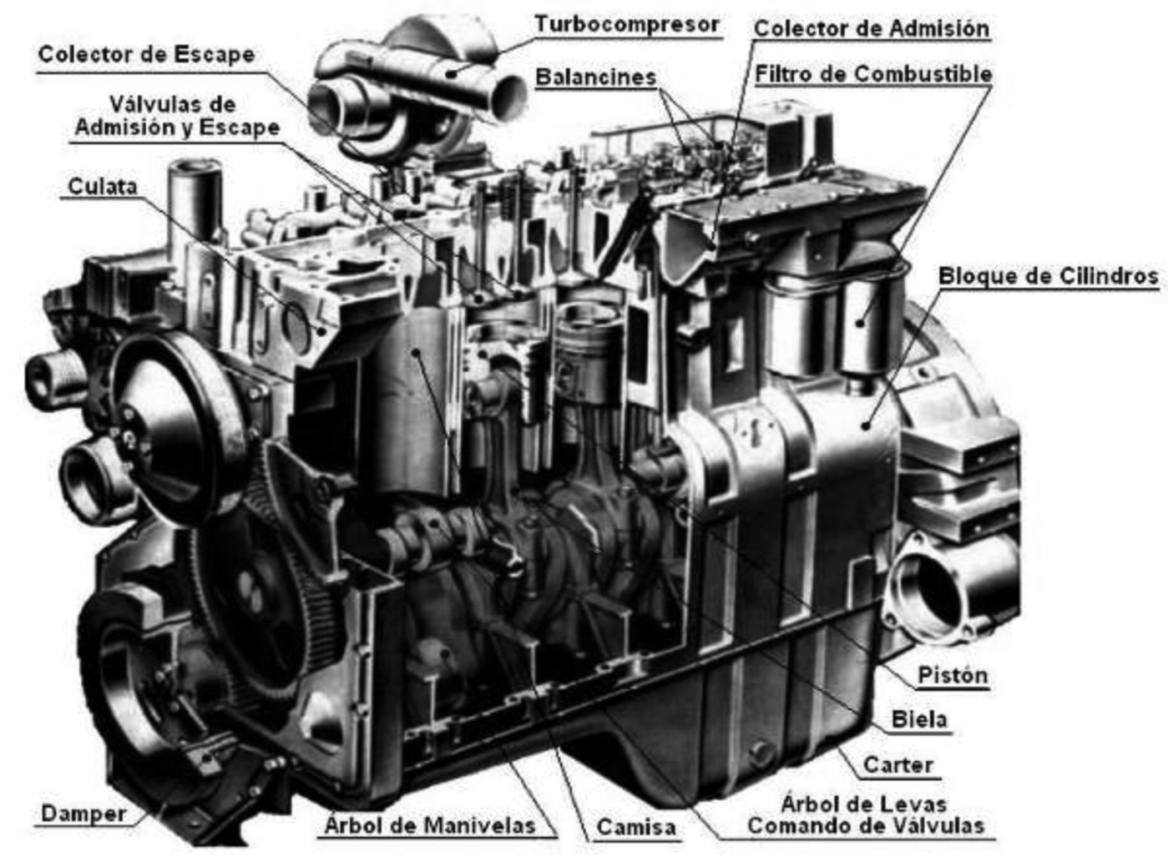

Figura 1. Motor ciclo diesel, Cofap ${ }^{[1]}$.

\section{Figure 1. Engine diesel, Cofap[1].}

Este conjunto está sometido a diferentes estados de cargas, debido a la expansión de los gases durante el proceso de la combustión. La figura 2 es una representación de esta situación.

Durante la combustión, la presión de los gases en algunas circunstancias puede alcanzar valores del orden de 200 bares en los motores diesel modernos. Gran parte de esa carga mecánica es transferida a los segmentos del pistón del primer canal, causando en las regiones próximas a la inversión del movimiento de esos segmentos una elevada solicitación tribológica. En algunos casos la aprobación o validación de un motor ciclo diesel impone la realización de ensayos cíclicos con duración de un mínimo 10 x $10^{8}$ ciclos.

En cada ciclo, los elevados niveles de presión y solicitación son transferidos a la estructura del motor, en particular las paredes resistentes del bloque junto al alojamiento de los cilindros, lo que hace necesario conocer la respuesta del hierro fundido a la solicitación mecánica, posibilitando así optimizar este material a cada aplicación a la cual será sometido.

La temperatura de la superficie de los cilindros puede alcanzar valores del orden de $150^{\circ} \mathrm{C}$ en regiones próximas al punto muerto superior de los seg-

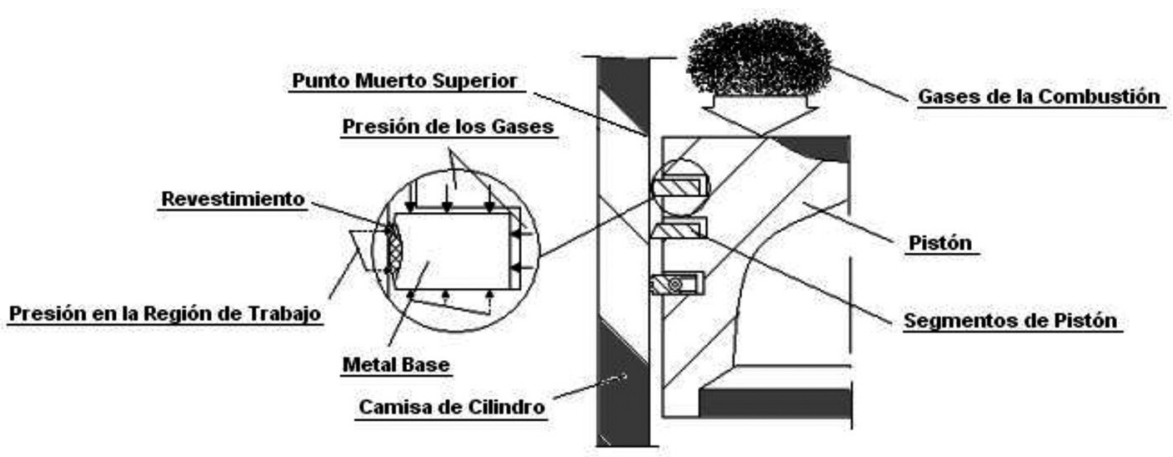

Figura 2. Esquema de funcionamiento del conjunto pistón-segmentoscamisa del cilindro, Vatavuk ${ }^{[2]}$.

Figure 2. Functional diagram of the piston-cylinder liner segments, Vatavuk ${ }^{[2]}$ 
mentos del primer canal, con un nivel de lubricación muy bajo impuesto por la actual legislación de emisiones de contaminantes atmosféricos.

En la realidad estas imposiciones están siendo cumplidas a través de técnicas especiales de acabado superficial de cilindros, exigiendo, además, que este acabado se mantenga durante mayores periodos de funcionamiento, lo que requiere el desarrollo de aleaciones de hierro fundido más resistentes al desgaste, que en el caso de los motores ciclo diesel están encuadradas generalmente en hierro fundido gris. Este material se constituye de una matriz ferrosa, envolviendo una elevada fracción volumétrica de grafito laminar, además de la presencia frecuente de un eutéctico rico en fósforo (esteadita).

La matriz más común de los hierros fundidos grises es la perlítica, constituida por láminas de ferrita y cementita. Un cambio de paradigma en términos de matriz de hierro fundido gris es la utilización de la estructura bainítica, que siendo bien tratada térmicamente puede constituir un interesante elemento para las camisas de cilindros de los motores de combustión interna de ciclo diesel. En el pasado reciente, la utilización de la estructura bainítica sin la realización del tratamiento térmico posterior a la fusión dio como resultado una inestabilidad dimensional que es evitable.

El presente estudio está enfocado a la medida de desgaste comparativo de los cilindros confeccionados con la aleación convencional con relación al hierro fundido gris de estructura bainítica, como respuesta en términos de desgaste, de los segmentos de pistón (mantenidos con estructura constante) que trabajaron en este conjunto experimental aplicados al motor estudiado.

\section{FUNDAMENTOS TEÓRICOS $Y$ REVISIÓN BIBLIOGRÁFICA. MECANISMOS DE DESGASTE}

Existen diversas clasificaciones para los mecanismos de desgaste; muchos de ellos están presentes en estos nudos tribológicos y serán analizados. Dependiendo del autor, el desgaste verificado en segmentos de pistón es descrito como "adhesivo" (B. Ruddy et al. ${ }^{[3]}$ ), "adhesión durante el asentamiento seguido de abrasión y corrosión durante el uso" (Barber \& Ludema ${ }^{[4]}$ ), o "micro-pulimentos" (Greenwood ${ }^{[5]}$ ). En este trabajo se decidió adoptar la nomenclatura definida por dos libros de texto recientes: Hutchings ${ }^{[6]}$ y Ludema ${ }^{[7]}$ "Desgaste por Deslizamiento". Aunque la adhesión ejerza un papel importante, existen otros procesos físicos y químicos actuando en el desgaste cuando dos cuerpos se deslizan entre sí. En el área automotora el término "desgaste adhesivo" también es asociado a la transferencia macroscópica de material entre el segmento y el cilindro (Demarchi ${ }^{[8]}$ ), o sea, a un desgaste bastante severo también denominado "engripamiento" o "scuffing".

El desgaste por deslizamiento es comúnmente modelizado por la ecuación de Archard, $\mathrm{Q}=\mathrm{K} . \mathrm{W} / \mathrm{H}\left(\mathrm{mm}^{3} / \mathrm{m}\right)$, donde:

Q: volumen desgastado del material más blando, por unidad de distancia recorrida. K: coeficiente adimensional de desgaste, W: carga normal [N] y $\mathrm{H}$ : dureza del cuerpo más blando $\left[\mathrm{Nm}^{-2}\right]$.

La ecuación de Archard es ampliamente usada por su simplicidad y también por permitir cuantificar la severidad del desgaste en diferentes sistemas por el coeficiente $\mathrm{K}$. El parámetro $\mathrm{k}=\mathrm{K} / \mathrm{H}\left[\mathrm{mm}^{3}(\mathrm{Nm})^{-1}\right]$ es más común en aplicaciones de ingeniería y es denominado coeficiente dimensional de desgaste. El coeficiente $\mathrm{k}$ representa el volumen en $\left[\mathrm{mm}^{3}\right]$ de material eliminado por desgaste por unidad de distancia recorrida $[\mathrm{m}]$ por unidad de carga normal en el contacto [N].

La superficie desgastada generalmente presenta aspecto pulido. Ya el desgaste severo produce partículas metálicas mucho mayores $(20$ a $200 \mu \mathrm{m})$ y, consecuentemente, la superficie tiene aspecto rugoso. Por su alta tasa de desgaste, esto es inaceptable en la mayoría de los sistemas mecánicos.

El desgaste abrasivo ocurre por la acción de partículas duras presionadas y deslizadas sobre las superficies. En motores de combustión interna las partículas que pueden provocar el desgaste abrasivo pueden tener diversos orígenes. Los principales son:

- Cenizas y material carbonizado, residuos sólidos de la combustión.

- Partículas metálicas y óxidos provenientes del desgaste.

- Polvo admitido junto con el aire y no bloqueado por el sistema de filtraje.

- Partículas metálicas oriundas de los procesos de fabricación, que permanecen en el motor por limpieza deficiente.

- Residuos de arena utilizados en la fundición del bloque y culatas, que permanecen en el motor por limpieza deficiente (Moore y Hutchings ${ }^{[6]}$ y Richardson ${ }^{[9]}$ ).

En motores de combustión interna, el desgaste corrosivo se debe a reacciones químicas (mezclas de aceite y combustible) en la cara de contacto entre el segmento y el cilindro. La presencia de azufre en el combustible aumenta el desgaste corrosivo. Aun con bajos porcentajes de azufre, otros ácidos se forman, como por ejemplo el ácido nítrico (Furuhama ${ }^{[10]}$ ). Se cree que el desgaste corrosivo es el mecanismo dominante cuando el motor opera muy frío o muy caliente (Behrens ${ }^{[11]}$ ). En particular, se nota un gran 
aumento en el desgaste del cilindro cuando la temperatura de la pared del mismo cae por debajo del punto de condensación de los productos de la combustión (Behrens ${ }^{[11]}$ ). La corrosión puede ser significativa en motores de combustión interna que funcionen con combustibles con alto grado de azufre.

Varios autores consideran el desgaste provocado por la fractura de las asperezas de la capa superficial por la acción de cargas alternativas, como un mecanismo independiente. Otros aun, solo consideran como desgaste por fatiga si las macro-tensiones envueltas son altas. Vatavuk ${ }^{[2]}$, dice que la caracterización de fatiga en la superficie de segmentos es difícil porque las macro-tensiones son muy bajas. Sin embargo, como se apreció durante el avance de este trabajo, las tensiones que actúan en las asperezas en contacto son bastante altas, y generalmente es acertado que los así llamados mecanismos de desgaste adhesivo y oxidativo ocurran, en ocasiones, como resultado de fracturas a lo largo de los puntos microscópicos de contacto (Martínez ${ }^{[12]}$ ). Otras teorías priorizan los aspectos mecánicos, más específicamente el aumento de la rugosidad provocado por deformación plástica. Ludema ${ }^{[13]}$ considera que la fatiga de bajo ciclo (régimen plástico) es la explicación más probable de que ocurra este desgaste.

\subsection{Caracterización del sistema tribológico}

Los segmentos de pistón, en especial el del primer canal, están sujetos a complejas y severas condiciones tribológicas:

- Velocidades medias de hasta $20 \mathrm{~m} / \mathrm{s}$. Como el movimiento es alternativo, la velocidad es variable, siendo cero en los puntos de reversión y máxima aproximadamente en la mitad del recorrido o trayectoria.

- Presiones de hasta $20 \mathrm{MPa}$ (200 bar) en algunos motores diesel de última generación. Esta presión varía a lo largo de los cuatro tiempos del motor.

- La pared del cilindro es en general de hierro fundido, con un típico acabado superficial de bruñido. La rugosidad superficial es del orden de $\mathrm{Rz}=6 \mu \mathrm{m}$ (Ra aproximadamente 1,0 ) cuando es nueva.

- La temperatura en la pared del cilindro es en torno de $100^{\circ} \mathrm{C}$, mientras que la del primer segmento es del orden de $250^{\circ} \mathrm{C}$.

- En consecuencia, la película de aceite lubricante está en el orden de algunos pocos micrómetros en el medio del recorrido, más, en el orden de apenas décimas de micrómetros, en los puntos de reversión, o sea: del mismo orden que la altura de las asperezas, y por tanto el régimen de lubricación es límite.
- El primer segmento, para motores diesel es en acero o hierro fundido nodular, con un revestimiento anti-desgaste. Este revestimiento es generalmente molibdeno, más específicamente una mezcla a base de molibdeno, níquel y cromo aplicada por plasma, o cromo duro depositado electrolíticamente.

\subsection{Métodos de medición de desgaste}

El método más común de medición del desgaste de segmentos es el aumento de holgura entre puntas "gap": se monta el segmento en un patrón con diámetro de agujero conocido y se mide la holgura entre las puntas del segmento con una lámina cónica graduada, con resolución de 0,05 mm. Comparando con el valor inicial, se tiene el aumento de holgura entre puntas. Ese valor puede ser entendido como la media de desgaste radial multiplicado por $2 \mathrm{~S}$, una vez que lo que es medido es la diferencia entre un perímetro conocido (patrón) y el perímetro del segmento. Es común caracterizar la tasa de desgaste, medida por el aumento de holgura entre puntas, en unidades como $\mu \mathrm{m} / 100 \mathrm{~h}$ o $\mu \mathrm{m} / 1000 \mathrm{Km}$. Como esta tasa acostumbra a disminuir con pruebas más largas, durante el asentamiento se produce mayor desgaste, por lo que es preciso tener cuidado al comparar valores provenientes de ensayos con diferentes duraciones. Otro método bastante común de medición de desgaste en segmentos es la variación del espesor radial. Se debe notar que, en general, el desgaste no es uniforme a lo largo del segmento, siendo mayor en las puntas. Shuster ${ }^{[14]}$ por ejemplo, verifica desgaste de $150 \%$ mayor en las puntas que la media a lo largo del resto del segmento.

En trabajos de desarrollo se acostumbra también a indicar el desgaste por la pérdida de masa. Aunque obtenida a través de una balanza micro-analítica con resolución de 0,1 mg, esa medida debe ser analizada con cuidado: el bajo desgaste verificado en segmentos (del orden de $10 \mathrm{mg} / 100 \mathrm{~h}$ ) puede inducir a conclusiones equivocadas como resultado de cualquier transferencia de material para el segmento.

\subsection{Desgaste de cilindros}

La medición del desgaste en el cilindro trae aún mayores dificultades. Medidas de la variación diametral se confunden con deformaciones geométricas que el cilindro sufre y son, en general, de poca utilidad, excepto en el caso de desgastes muy grandes (del orden de varias decenas de micrómetros). Debe des- 
tacarse que un aumento de $100 \mu \mathrm{m}$ en el diámetro del cilindro implica directamente un aumento de la holgura funcional entre puntas del segmento $S$ veces mayor (aprox. $300 \mu \mathrm{m}$ ). Así, una medida usual en la "práctica de taller" es una medición de la holgura entre puntas del segmento una vez montado en el cilindro.

El cilindro presenta desgaste diferenciado para los diversos puntos de su diámetro: mayor en el punto muerto superior (PMS) del primer segmento, (Barber \& Ludema ${ }^{[4]}$ ), ya que esa región, como se vio, sufre las condiciones tribológicas más severas. Como el desgaste del cilindro es bastante pequeño, una medida usual, de más difícil interpretación, es la variación de la rugosidad (Shuster ${ }^{[14]}$ ). El desgaste de segmentos depende fuertemente del ciclo de ensayos (Thom ${ }^{[15]}$ ). En cilindros de hierro fundido, el mecanismo de desgaste predominante parece ser el del pulido, (Thom $\left.{ }^{[15]}\right)$ : el desgaste parece que ocurre gradual y uniformemente y define el pulido como la eliminación progresiva del material de la superficie por la acción de la fricción de otro cuerpo bajo condiciones en que el material es eliminado sin huellas visibles, fracturas o deformación plástica.

\subsection{Realización de los ensayos}

Para la realización de los ensayos fueron siempre tomadas seis muestras, despreciando aquélla que más se apartaba de la media aritmética obtenida, por lo que fueron valoradas cinco. En todos los casos el error estuvo por debajo de los admisibles en trabajos de ingeniería (10\%).

\subsubsection{Determinación y prueba de los materiales utilizados}

\section{Composición química y estructura:}

El material propuesto, denominado material B, fue producido a partir de una aleación de composición química presentada en la tabla I.
El examen de la tabla anterior indica la presencia de los elementos químicos comunes a los hierros fundidos grises como carbono y silicio así como la presencia de molibdeno y níquel en busca de la obtención de una estructura bainítica de fundición. El material propuesto tuvo también en su composición química elementos de aleaciones formadores de carburos del tipo MC (niobio, vanadio y titanio), con la finalidad de aumentar la resistencia al desgaste, como fue recomendado en trabajos anteriores para camisas basadas en estructura perlítica (Vatavuk ${ }^{[2]}$ ). La grafitización obtenida en la superficie de trabajo con los segmentos de pistón es predominantemente del tipo D y E, tamaño 5, según norma ASTM A 247. La estructura bainítica obtenida puede apreciarse en la figura 3.

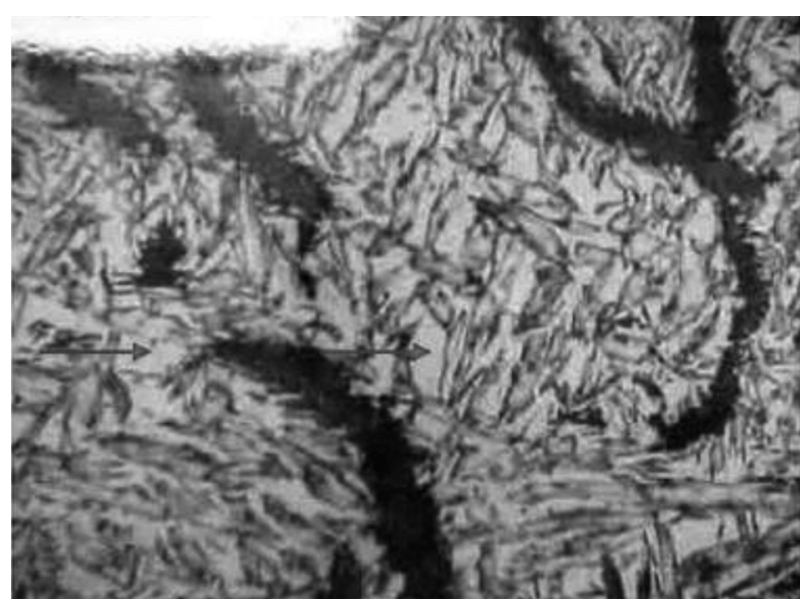

Figura 3. Micro-estructura constituida por bainita superior. Las flechas indican la austenita estabilizada (x1000). Ataque Nital.

Figure 3. Micro-structure consisting of upper bainite. The arrows indicate the stabilized austenite (x1000). Attack Nital.

La adición de elementos de aleación formadores de carburos de tipo MC, niobio, titanio y vanadio, promovió la precipitación de carburos que puede ser observada en la figura 4.

Tabla I. Composición química media del material propuesto B (\% en peso)

Table I. Average chemical composition of the proposed material B (\% in weight)

\begin{tabular}{cccccccccccc}
\hline Ceq & $\mathbf{C}$ & $\mathbf{S i}$ & $\mathbf{M n}$ & $\mathbf{P}$ & $\mathbf{S}$ & $\mathbf{C u}$ & $\mathbf{C r}$ & $\mathbf{N i}$ & $\mathrm{Mo}$ & $\mathbf{V}$ & $\mathbf{N b}$ \\
\hline 3,520 & 2,900 & 1,820 & 0,180 & 0,037 & 0,017 & 0,063 & 0,031 & 1,460 & 1,320 & 0,155 & 0,170 \\
\hline
\end{tabular}




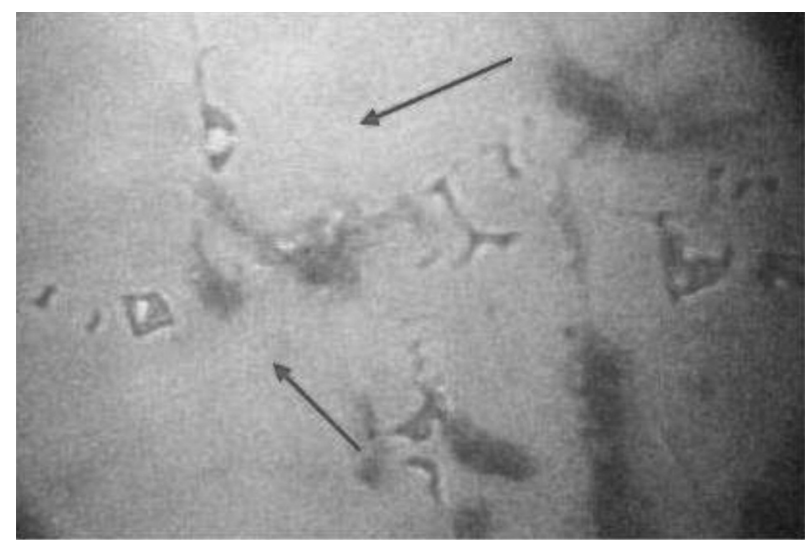

Figura 4. Las flechas indican los carburos tipo MC (x1000). Sin ataque.

Figure 4. The arrows indicate the MC type carbides (x1000). Without attack.

En el trabajo fueron realizados tratamientos isotérmicos con determinación de la dureza, con el fin de conseguir la mejor temperatura para condiciones de fabricación del componente. Las temperaturas escogidas y el ciclo de tiempo de tratamiento pueden ser visualizados en el gráfico de la figura 5. La temperatura de tratamiento utilizada en este estudio fue de $680^{\circ} \mathrm{C}$, y la variación máxima fue de $10^{\circ} \mathrm{C}$.

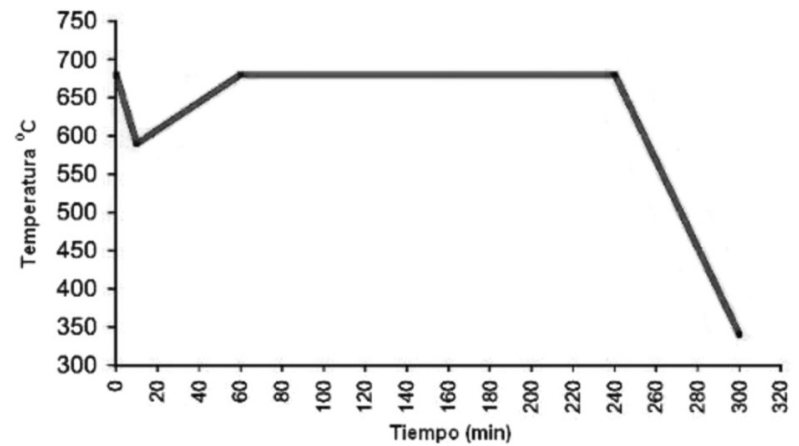

Figura 5. Ciclo de tratamiento térmico en horno de inducción.

Figure 5. Heat treatment cycle induction furnace.
El ciclo observado en la figura anterior muestra inicialmente un enfriamiento, que ocurre debido a la carga inicial del horno con la masa relativamente elevada de camisas (cincuenta piezas) pesando cada una aproximadamente tres kilogramos.

Con la finalidad de estabilizar la estructura para aplicaciones de camisas de cilindro, fueron realizados tratamientos isotérmicos (a las temperaturas apuntadas en la técnica experimental), produciendo microestructuras típicas como la observada en la figura 6.

El material con el cual se estableció la comparación de la aleación propuesta fue de un hierro fundido gris de matriz perlítica, considerado de amplia utilidad cuando se piensa en la resistencia al desgaste. Para este estudio fue denominado material P.

La composición química de esta aleación está representada en la tabla II, la cual constituye una composición química típica de hierros fundidos grises. La grafitización de este componente se encuadra como predominantemente del tipo A y tamaño 4 y 6, conforme ASTM A 247. El proceso de fundición utilizado fue el centrífugo para las dos aleaciones, manteniéndose las mismas condiciones de ciclo y rotación de la máquina.

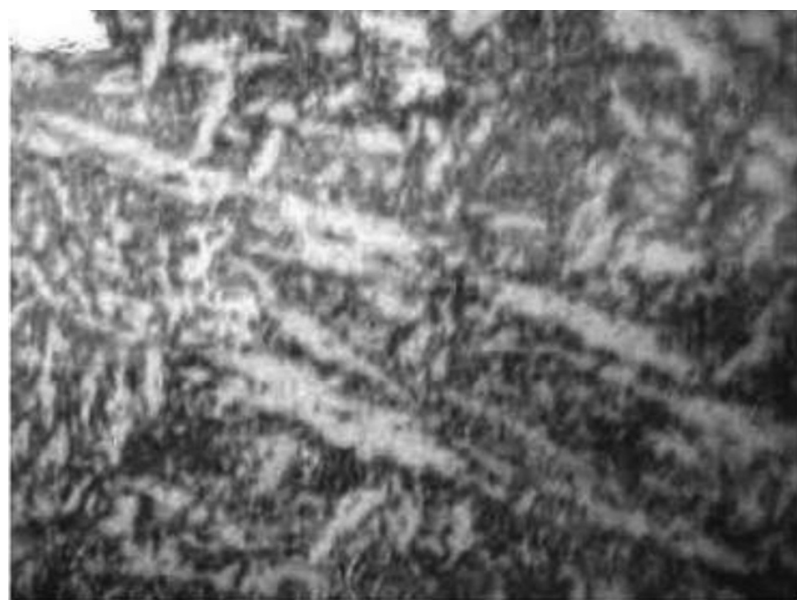

Figura 6. Material revenido a $680^{\circ} \mathrm{C}$ durante $3 \mathrm{~h}$ (x1000), matriz bainítica. Ataque Nital.

Figure 6. Material tempering at $680^{\circ} \mathrm{C}$ for $3 \mathrm{~h}$ (x1000), bainitic matrix. Attack Nital.

Tabla II. Composición química media del material base P (\% en peso)

Table II. Average chemical composition of the base material $P$ (\% in weight)

\begin{tabular}{cccccccc}
\hline Ceq & $\mathbf{C}$ & $\mathbf{S i}$ & $\mathbf{M n}$ & $\mathbf{P}$ & $\mathbf{S}$ & $\mathbf{C u}$ & $\mathbf{C r}$ \\
\hline 4,200 & 3,450 & 2,250 & 1,650 & 1,520 & 1,120 & 1,000 & 0,800 \\
\hline
\end{tabular}




\section{Propiedades mecánicas:}

Tanto el material propuesto como el convencional fueron sometidos a pruebas de dureza utilizando la escala Brinell, en condiciones para cargas de $188 \mathrm{kgf}$ y esferas de 2,5 mm de diámetro: para el material B, la dureza fue del orden de $290 \mathrm{HB}$ y en el caso del material perlítico $\mathrm{P}$, la dureza resultó ser de aproximadamente 250 HB. Para las dos aleaciones las medidas de dureza presentaron una desviación inferior a $5 \%$ de la media.

También fueron extraídos cuerpos de prueba para ensayos de tracción obedeciendo la norma ASTM A370, con la finalidad de determinar la resistencia a la tracción del material convencional y de la aleación propuesta, estableciendo así una comparación entre las dos. La tabla III presenta los valores de resistencia obtenidos en los cuerpos de prueba del material propuesto y convencional, con matrices bainítica y perlítica.

\section{Ensayo dinamométrico:}

Las camisas de cilindro, con el material propuesto y el convencional, fueron ensayadas utilizándose un motor de ciclo diesel Scania de seis cilindros en línea, con potencia de $150 \mathrm{kw}$, obedeciendo a un montaje a partir de la bomba de agua, que redujese diferencias en términos de solicitación. Hay que resaltar que una de las piezas con material propuesto fue instalada en la posición más distante de la bomba de agua, cuidando las condiciones tribológicas de solicitación en el motor, de manera que cuanto mayor es la distancia de la bomba de agua, teóricamente mayor será la temperatura del líquido de enfriamiento y consecuentemente, la de la camisa de cilindro. El ensayo dinamométrico realizado fue a plena carga, ya que este motor originalmente es destinado a aplicación estacionaria, con crecimiento de potencia de $30 \%$ $(195 \mathrm{~kW})$, conseguido a través de un mayor torque, para la misma rotación en el ensayo dinamométrico, durante $500 \mathrm{~h}$.

Con la finalidad de conseguir determinaciones del desgaste en los cilindros, que de una manera general precisan de pruebas muy largas para presentar desgaste apreciable, se aumentó la solicitación tribológica a través del uso de aceite lubricante de baja eficiencia (especificado por el fabricante según la denominación SAE 15W40), además del combustible de baja calidad, con diesel con el mayor contenido de azufre disponible en el mercado (0,5\% máximo).

Tabla III. Resultados de ensayos de tracción para las muestras propuesta y convencional. El valor marcado en negrita fue rechazado para calcular la media

Table III. Results of tensile tests for the samples standard and proposal. The bold value was rejected for averaging

\begin{tabular}{lccc}
\hline Muestra & Material & $\begin{array}{c}\text { Resistencia } \\
\text { a tracción, MPa }\end{array}$ & MEDIA, MPa \\
\hline B1 & 320 & \\
B2 & 293 & PERLÍTICO \\
B3 & 305 & \\
B4 & PERLÍTICO & $\mathbf{2 8 8}$ & \\
B5 & 308 & \\
B6 & 308 & \\
\hline MEDIA & & 1.534 & \\
\hline P1 & 507 & \\
P2 & 505 & \\
P3 & 509 & \\
P4 & 477 & \\
P5 & & 494 & 491 \\
P6 & BAINÍTICO & 470 & \\
\hline MEDIA & $\mathbf{4 6 7}$ \\
\hline
\end{tabular}


MEJORAS EN LA CONSTRUCCIÓN DE CAMISAS DE CILINDRO DE MOTORES DE COMBUSTIÓN INTERNA CICLO DIESEL IMPROVEMENT IN THE PRODUCTION OF CYLINDER SHIRT OF INNER DIESEL COMBUSTIÓN ENGINES

\section{Cálculo de desgaste de los elementos del sistema:}

Para las camisas de cilindro, se utilizó el criterio de área pulida La determinación del porcentaje de área pulida se llevó a cabo siguiendo la metodología del fabricante de las camisas de cilindro (Cofap $\left.{ }^{[1]}\right)$. El porcentaje de área pulida se obtuvo conforme con la siguiente expresión (Ec. (1)):

$$
\mathrm{AP} \%=\frac{\text { Área Media }}{S \cdot \varnothing \cdot \mathrm{L}}
$$

donde:

AP $\%=$ Porcentaje del área pulida medida.

Área medida $=$ Área medida por la metodología expuesta anteriormente.

$\varnothing=$ Diámetro interno de la camisa de cilindro.

$\mathrm{L}=$ Distancia entre el PMI del segmento de aceite y el PMS del segmento de comprensión.

El desgaste de los segmentos de pistón fue obtenido, primeramente, midiendo las dimensiones iniciales como el espesor radial antes y después de la realización de la prueba con la utilización de un pie de rey (Nigro $\left.{ }^{[16]}\right)$. La diferencia corresponde al desgaste en el espesor radial. Otra medida de desgaste empleada fue la holgura entre puntas, también con la utilización de un pie de rey. Para este caso se determinó la holgura entre las puntas inicialmente y después de la realización de la prueba dinamométrica. Teóricamente la medida de variación de holgura entre puntas después de la prueba debe guardar relación con la variación del espesor radial según la siguiente expresión (Ec. (2)):

$$
\triangle \mathrm{GAP}=2 \cdot S \cdot \Delta \mathrm{RAD}
$$

donde:

$\triangle \mathrm{GAP}=$ Variación de la holgura entre puntas.

$\triangle \mathrm{RAD}=$ Variación del espesor radial .

\section{Resultados según el área pulida:}

Los resultados obtenidos de las mediciones del área pulida, real y aparente para las camisas con el material propuesto bainítico $\mathrm{B}$ y el convencional perlítico $\mathrm{P}$, se muestran en la tabla IV.

Los valores presentados en la tabla corresponden a la media para cinco cilindros de cada material.

El examen de la tabla IV indica una apreciable diferencia en términos de desgaste presentando el
Tabla IV. Dimensiones de la área pulida (\%)

Table IV. Dimensions of the polished area (\%)

\begin{tabular}{lcc}
\hline Áreas & B & P \\
\hline Pulida real (media) & 3,33 & 11,00 \\
Pulida aparente (media) & 9,33 & 25,33 \\
\hline
\end{tabular}

material bainítico B mejores resultados, lo que confirma su capacidad de mantener el motor en mejores condiciones de funcionamiento

\section{Resultados de la medición de circularidad y cilindricidad:}

Al final de 500 h de prueba, después de extraer las camisas, éstas fueron sometidas a una serie de ensayos metrológicos, como circularidad y cilindricidad, que corresponden a importantes medidas geométricas en el funcionamiento global de los sistemas cilindro, pistón y segmentos de pistón, en lo que se refiere al consumo de aceite lubricante y al paso de gases de la cámara de combustión para el cárter, que deben ser mantenidos en valores mínimos, según se exige para leyes de emisiones contaminantes.

La tabla $\mathrm{V}$ presenta estos resultados, a través de los cuales se puede concluir que el material propuesto se comportó un poco mejor que la aleación convencional.

\section{Resultados en la variación de la rugosidad:}

En la tabla VI se puede observar la variación de Ra y en la tabla VII, la de Rz.

\section{Valores de desgaste en los segmentos (aros):}

La tabla VIII muestra los valores de desgaste del conjunto de segmentos que trabajaron con los tres cilindros convencionales y los tres del material propuesto.

El examen de la tabla VIII muestra que el desgaste del conjunto de segmentos que trabajaron con el material convencional y el propuesto es similar, lo 
Tabla V. Resultados de circularidad y cilindricidad

Table V. Results of circularity and cylindricity

\begin{tabular}{|c|c|c|c|c|c|c|c|c|c|}
\hline \multirow[b]{2}{*}{ Cilindro } & \multirow{2}{*}{ Camisa } & \multirow{2}{*}{ Material } & \multirow{2}{*}{ Horas } & \multicolumn{3}{|c|}{ Cilindricidad } & \multicolumn{3}{|c|}{ Circularidad } \\
\hline & & & & $\begin{array}{c}\text { Antes } \\
(\mu \mathrm{m})\end{array}$ & $\begin{array}{c}\text { Después } \\
(\mu \mathrm{m})\end{array}$ & $\begin{array}{l}\text { Diferencia } \\
(\%)\end{array}$ & $\begin{array}{c}\text { Antes } \\
(\mu \mathrm{m})\end{array}$ & $\begin{array}{c}\text { Después } \\
(\mu \mathrm{m})\end{array}$ & $\begin{array}{c}\text { Diferencia } \\
(\%)\end{array}$ \\
\hline 1 & 1B & B & & 3,68 & 12,51 & 71 & 2,26 & 9,05 & 75 \\
\hline 2 & $2 \mathrm{P}$ & $P$ & & 2,86 & 20,82 & 86 & 1,93 & 14,31 & 87 \\
\hline 3 & $3 B$ & B & & 3,69 & 18,36 & 80 & 1,68 & 11,85 & 86 \\
\hline 4 & $4 \mathrm{P}$ & $\mathrm{P}$ & & 2,83 & 14,73 & 81 & 1,64 & 15,85 & 90 \\
\hline 5 & $5 B$ & B & 500 & 3,85 & 13,14 & 71 & 1,42 & 9,41 & 85 \\
\hline 5 & $6 \mathrm{P}$ & $\mathrm{P}$ & & 5,15 & 18,12 & 72 & 1,27 & 11,49 & 89 \\
\hline
\end{tabular}

Tabla VI. Resultados de la variación de la rugosidad a través del parámetro de Ra

Table VI. Results of the variation of the roughness by parameter Ra

\begin{tabular}{|c|c|c|c|c|c|c|}
\hline & \multirow[b]{2}{*}{ Camisa } & \multirow[b]{2}{*}{ Material } & \multirow[b]{2}{*}{ Horas } & \multicolumn{3}{|c|}{$\mathbf{R a}$} \\
\hline & & & & $\begin{array}{c}\text { Antes } \\
(\mu \mathrm{m})\end{array}$ & $\begin{array}{l}\text { Después } \\
(\mu \mathrm{m})\end{array}$ & $\begin{array}{c}\text { Diferencia } \\
(\%)\end{array}$ \\
\hline 1 & 1B & B & & 0,774 & 0,476 & 39 \\
\hline 2 & $2 \mathrm{P}$ & $P$ & & 0,861 & 0,432 & 50 \\
\hline 3 & $3 B$ & B & 500 & 0,894 & 0,463 & 48 \\
\hline 4 & $4 \mathrm{P}$ & $P$ & & 0,966 & 0,407 & 58 \\
\hline 5 & $5 B$ & $B$ & & 0,936 & 0,537 & 43 \\
\hline 5 & $6 P$ & $P$ & & 1,004 & 0,452 & 55 \\
\hline
\end{tabular}

Tabla VII. Resultados de la variación de la rugosidad a través del parámetro de $\mathrm{Rz}$

Table VII. Results of the variation of the roughness by parameter Rz

\begin{tabular}{|c|c|c|c|c|c|c|}
\hline \multirow[b]{2}{*}{ Cilindro } & \multirow[b]{2}{*}{ Camisa } & \multirow[b]{2}{*}{ Material } & \multirow[b]{2}{*}{ Horas } & \multicolumn{3}{|c|}{$\mathbf{R z}$} \\
\hline & & & & $\begin{array}{c}\text { Antes } \\
(\mu \mathrm{m})\end{array}$ & $\begin{array}{l}\text { Después } \\
(\mu \mathrm{m})\end{array}$ & $\begin{array}{c}\text { Diferencia } \\
(\%)\end{array}$ \\
\hline 1 & $1 \mathrm{~B}$ & B & & 3,518 & 2,236 & 36 \\
\hline 2 & $2 \mathrm{P}$ & $P$ & & 3,964 & 2,008 & 49 \\
\hline 3 & $3 B$ & $B$ & 500 & 4,245 & 2,121 & 50 \\
\hline 4 & $4 \mathrm{P}$ & $P$ & & 4,268 & 2,097 & 51 \\
\hline 5 & $5 B$ & B & & 4,093 & 2,627 & 36 \\
\hline 5 & $6 P$ & $P$ & & 4,549 & 2,289 & 50 \\
\hline
\end{tabular}


MEJORAS EN LA CONSTRUCCIÓN DE CAMISAS DE CILINDRO DE MOTORES DE COMBUSTIÓN INTERNA CICLO DIESEL IMPROVEMENT IN THE PRODUCTION OF CYLINDER SHIRT OF INNER DIESEL COMBUSTIÓN ENGINES

Tabla VIII. Dimensiones de los segmentos de pistón ensayados

$(\mathrm{mm})$

Table VIII. Dimensions of the piston segments tested $(\mathrm{mm})$

\begin{tabular}{lcccccc}
\hline & \multicolumn{2}{c}{$\mathbf{1}^{\mathbf{0}}$ Canal } & \multicolumn{2}{c}{$\mathbf{2}^{\mathbf{2}}$ Canal } & \multicolumn{2}{c}{$\mathbf{3}^{\mathbf{0}}$ Canal } \\
\cline { 2 - 7 } Cilindro & $\mathbf{\Delta G A P}$ & $\mathbf{\Delta R A D}$ & $\mathbf{\Delta G A P}$ & $\mathbf{\Delta R A D}$ & $\mathbf{\Delta G A P}$ & $\mathbf{\Delta R A D}$ \\
\hline 1B & 0,04 & 0,012 & 0,05 & 0,014 & 0,05 & 0,010 \\
2P & 0,04 & 0,014 & 0,02 & 0,008 & 0,05 & 0,06 \\
3B & 0,05 & 0,016 & 0,08 & 0,0017 & 0,09 & 0,008 \\
4P & 0,05 & 0,017 & 0,09 & 0,0017 & 0,08 & 0,010 \\
5B & 0,08 & 0,015 & 0,10 & 0,024 & 0,08 & 0,012 \\
6P & 0,06 & 0,017 & 0,09 & 0,022 & 0,11 & 0,014 \\
\hline
\end{tabular}

que permite decir que el conjunto segmentos/cilindro lleva ventaja en términos de desgaste cuando se utiliza la aleación propuesta, pudiendo así satisfacer las condiciones severas impuestas por las modernas leyes de emisiones.

\section{CONCLUSIONES}

El conjunto de resultados reunidos en el presente estudio permite llegar a las siguientes conclusiones:

- La estructura fundida de la aleación propuesta es bainítica atendiendo los requisitos existentes en el trabajo, indicando el efecto de la adición de níquel y principalmente de molibdeno.

- El tratamiento isotérmico a $680^{\circ} \mathrm{C}$ produjo cambios en la estructura bainítica, con completa descomposición de la austenita retenida, por lo menos al nivel de inspección empleado en este estudio.

- El desgaste de los cilindros estimado por el área pulida fue de 3,33\% para el material propuesto y de $11,00 \%$ para la aleación convencional.

- El desgaste de los cilindros estimado de área pulida aparente fue de 9,33\% para el material propuesto y de $25,33 \%$ para la aleación convencional.

- La variación de la rugosidad de los cilindros a $30 \mathrm{~mm}$ del punto muerto superior de los segmentos del primer canal del pistón, no presentó diferencias sensibles entre los dos materiales utilizados.

- El desgaste de los segmentos de pistón que trabajaron con el material propuesto y la aleación convencional fue similar.
- El desgaste combinado entre segmentos y cilindro fue menor en el conjunto que trabajó con el material propuesto, aun posicionando éste último más distante de la bomba de agua.

\section{REFERENCIAS}

[1] Cofap-CIA, Informe Técnico, Fabrica de Peças, Critério de determinação de área polida. Apostila, Santo André, Brasil, 1989.

[2] J. Vatavuk, Tese (Doutorado), Escola Politécnica - Universida de Sao Paolo, Brasil, 1994.

[3] B. Ruddy. Leeds/Lyon Symposium on Tribology, 1982, pp.162-169.

[4] G. Barber y K. Ludema, Wear 100 (1982) 315331.

[5] J.A. Greenwood y J. Williamson, "Contact of nominally flat surfaces". Proc. R. Soc. London, Series A, Vol. 295, 1966, pp.300-319.

[6] I.M. Hutchings, "Tribology: Friction and Wear of Engineering Materials", Edward Arnold, London, 1992.

[7] K. Ludema, "Friction, Wear, Lubrication - A Textbook in Tribology", CRC Press. Boca Raton, 1996.

[8] V. Demarchi, Dissertação de Mestrado, Escola Politécnica da Universidade de São Paulo, Brasil, 1994.

[9] D. Richardson y S. Krause, Proc. of the 3rd Ricardo Software, International Conference, Detroit, EE.UU., 1998.

[10] S. Furuhama, M. Hiruma y M. Tsuzita, "An Increase of Engine Oil Consumption at High Temperature of Piston and Cylinder", SAE Review 810976, 1991, pp. 92-1.010.

[11] R. Behrens, "Corrosion in Heavy Fuel Diesel 
Engines", Handbook of Analytical Methods for Materials, Vol. 49, 1998, pp. 479-485.

[12] F. Martínez, Tribología Integral, Ed. Noriega, México, 2010.

[13] K. Ludema, Wear 100 (1984) 315-331.

[14] Shuster, Met. al. Tribol. Revista Corrosión y Protección 42 (1999) 116-125.
[15] R. Thom, "Extended Oil Drain Intervals: Conservation of Resources or Reduction of Engine Life”. Informe SAE 951035, 1995.

[16] E.B. Nigro, Dissertação de Mestrado, Escola Politécnica da Universidade de São Paulo, Brasil, 1999. 